\title{
CONCEPTOS FUNDAMENTALES DEL FÚTBOL Y SU DESIGNACIÓN EN LAS CRÓNICAS DEPORTIVAS DEL REAL MADRID EN EL DIARIO MARCA
}

Resumen. El artículo presenta un análisis léxico de un corpus de 18 crónicas futbolísticas de partidos de fútbol del Real Madrid aparecidos en la web del diario Marca. El análisis de frecuencias de las palabras con significado léxico permite determinar los conceptos principales que funcionan como hilo conductor de las crónicas. Posteriormente el escrutinio de las concordancias en este corpus sirve para mostrar la variación léxica en torno a estos conceptos y los valores semánticos de las designaciones de estos conceptos.

Palabras claves: lexicología, semántica, lenguaje futbolístico, concepto, designación.

\section{El léxico futbolístico}

El nivel léxico es, sin duda, el más característico del lenguaje deportivo. Según Hernández Alonso (2003: 57), el 60\% del léxico de la prensa deportiva se puede considerar común o estándar, en tanto que el $40 \%$ tiene una particularidad deportiva propia. El léxico propiamente deportivo está compuesto por un $7 \%$ de léxico argótico, un $12 \%$ de léxico técnico-especializado y un $21 \%$ de léxico trasladado. Tratándose del fútbol, estos tres grupos de léxico deportivo se caracterizan del siguiente modo (Hernández Alonso, 2003: 58 y ss.):

1. Léxico argótico: Son las palabras y expresiones propias del fútbol, empleadas por los deportistas y los periodistas, muchos de cuyos términos propios tienen ya «una clara penetración en el español coloquial» (Naranjo de Arcos, 2011: 94), y que se han trasladado y se utilizan ya en otros ámbitos de la vida social, dada la popularidad de este deporte. Entre estos términos se cuentan: «hacer un caño (introducir el balón entre las piernas

\footnotetext{
* Uniwersytet Łódzki.
} 
del contrario para librarse de él y continuar la carrera), tocar (pasar la pelota con suavidad al compañero), gol cantado (fallar una ocasión muy clara) y vacas sagradas (expresión para referirse a los jugadores estrella del equipo)» (Álvarez Pedrosa, 2017: 60).

2. Léxico técnico-especializado: Se trata de un léxico especializado, de carácter internacional, cuyo origen se halla en la reglamentación del deporte. En este léxico predomina la denominación y la monosemia, con grandes aportaciones de neologismos y préstamos. Los manuales de estilo periodístico recomiendan no hacer un uso excesivo de los mismos para evitar la confusión e incomprensión del texto por parte de los lectores no iniciados. En el fútbol son tecnicismos: driblar, libre directo, desmarque, saque de banda, fuera de juego, etc.

3. Léxico trasladado: Es el léxico más empleado en las crónicas deportivas. Por medio de los usos trasladados, palabras habitualmente empleadas en campos ajenos al deporte (guerra, ciencia, técnica, religión, sabiduría popular, etc.) se trasladan al campo deportivo. Los principales recursos de traslación son la metáfora (el más utilizado), la sinécdoque, la polisemia y la sinonimia.

\section{Metodología}

Este artículo tiene como objetivo general realizar un análisis léxicosemántico de las crónicas deportivas del Real Madrid para identificar los conceptos recurrentes en estas crónicas en relación con el Real Madrid y con el deporte del fútbol. Ello servirá para un análisis posterior de la expresión léxica de estos conceptos y de cómo significan. A tal fin hemos escogido 18 crónicas de partidos de fútbol del Real Madrid, publicadas en la página web del diario Marca, entre las temporadas 2009/2010 y 2017/2018, recogidas en la hemeroteca de esta web. Para cada temporada se han elegido al azar un encuentro de la primera vuelta y otro de la segunda vuelta de la competición.

En el periodo estudiado, el sitio de internet del diario Marca fue la web de información deportiva más visitada en España, pasando de 3.089.000 visitantes diarios en la $3^{\text {a }}$ ola de 2009 del Estudio General de Medios (E.G.M.) de octubre-noviembre a 4.638 .000 visitantes en la $1^{\text {a }}$ ola de 2018 del E.G.M. de febrero-marzo. Por lo tanto, es el sitio web de referencia de la información deportiva en España.

En cuanto al Real Madrid, se trata del club de fútbol más laureado de España, con un palmarés en el que destaca el mayor número de campeonatos 
de liga nacional ganados, 33 hasta 2018, y el mayor número de entorchados continentales, con 13 copas de Europa o liga de campeones. Por ello ha sido galardonado como el Mejor Club Europeo y Mundial del siglo XX por la Federación Internacional de Historia y Estadística de Fútbol (IFFHS). Es, sin lugar a dudas, un club de influencia universal, con seguidores en todo el mundo y que mantiene una rivalidad social y deportiva con el F.C. Barcelona. Los 18 textos analizados corresponden a las siguientes crónicas y partidos:

Tabla 1. Corpus de crónicas deportivas analizadas

\begin{tabular}{|c|c|c|}
\hline Fecha & Partido y resultado & Titular de la crónica \\
\hline 2009.09.26 & Real Madrid - C. D. Tenerife: 3-0 & Excelencia en el remate \\
\hline 2010.04.24 & Real Zaragoza - Real Madrid: 1-2 & Kaká mantiene la fe \\
\hline 2010.11.20 & Real Madrid - Ath. Bilbao: 5-1 & El Real Madrid embiste de nuevo \\
\hline 2011.03.06 & $\begin{array}{l}\text { Racing de Santander - Real Madrid: } \\
1-3\end{array}$ & Özil ilumina al Madrid \\
\hline 2011.12.18 & Sevilla F.C. - Real Madrid: 2-6 & Pegada de líder \\
\hline 2012.04.21 & F.C. Barcelona - Real Madrid: 1-2 & La Liga en Cristiano \\
\hline 2012.12.16 & Real Madrid - R.C.D. Espanyol: 2-2 & El Espanyol deja señalado a Mou \\
\hline 2013.03.16 & Real Madrid - R.C.D. Mallorca: 5-2 & Demolition Team \\
\hline 2013.09.22 & Real Madrid - Getafe C.F.: 4-1 & A falta de Bale, Cristiano \\
\hline 2014.05.04 & Real Madrid - Valencia C.F.: 2-2 & Alves frustra al Madrid \\
\hline 2014.09.20 & R.C.D. La Coruña - Real Madrid: 2-8 & Más chulo que un ocho \\
\hline 2015.03.15 & Real Madrid - Levante U.D.: 2-0 & Clásico a punto \\
\hline 2015.12.20 & Real Madrid - Rayo Vallecano: 10-2 & Goles no son amores \\
\hline 2016.02.07 & Granada C.F: - Real Madrid: 1-2 & Modric es el clavo ardiendo \\
\hline 2016.11.19 & Atlético de Madrid - Real Madrid: 0-3 & Cristiano cierra el Calderón \\
\hline 2017.03.04 & S.D. Éibar - Real Madrid: $1-4$ & El Benzema que todos quieren \\
\hline 2017.03.04 & Real Madrid - Málaga C.F.: 3-2 & Un Madrid de rebajas \\
\hline 2018.02.10 & Real Madrid - Real Sociedad: 5-2 & El Madrid se afila \\
\hline
\end{tabular}

Fuente: elaboración propia. 
La herramienta utilizada para el análisis léxico ha sido el programa AntConc, el cual proporciona una lista con las frecuencias léxicas de los textos sin lematizar y que permite encontrar las concordancias de palabras concretas en el corpus textual. Hemos restringido el estudio a las palabras «que significan», esto es, las palabras con significado léxico autónomo, independiente de un contexto, y que pueden constituir núcleo del sintagma: sustantivos, adjetivos, verbos y adverbios (Ulrich, 2002). El resto de palabras, con significado gramatical, no se ha contabilizado.

\section{El contenido léxico-semántico de las crónicas del Real Madrid}

El análisis cuantitativo del léxico de las 18 crónicas muestreadas ofrece un total de 5.966 palabras gráficas (word tokens) y 2.704 tipos léxicos (word types). La tabla siguiente muestra las 30 palabras con significado léxico más frecuentes del texto.

Tabla 2. Palabras con significado léxico más frecuentes en el corpus analizado

\begin{tabular}{|c|c|l|c|c|l|c|c|l|}
\hline Núm. & Frec. & Palabra & Núm. & Frec. & Palabra & Núm. & Frec. & Palabra \\
\hline 1 & 181 & Madrid & 11 & 28 & minutos & 21 & 19 & Kaká \\
\hline 2 & 66 & Cristiano & 12 & 27 & goles & 22 & 19 & parte \\
\hline 3 & 66 & partido & 13 & 27 & liga & 23 & 18 & Ronaldo \\
\hline 4 & 59 & equipo & 14 & 22 & Bale & 24 & 17 & falta \\
\hline 5 & 50 & real & 15 & 22 & balón & 25 & 17 & di María \\
\hline 6 & 47 & gol & 16 & 22 & tiempo & 26 & 16 & descanso \\
\hline 7 & 44 & Benzema & 17 & 21 & mejor & 27 & 15 & centro \\
\hline 8 & 36 & Bernabéu & 18 & 20 & defensa & 28 & 15 & penalti \\
\hline 9 & 32 & hizo & 19 & 20 & Modric & 29 & 15 & volvió \\
\hline 10 & 29 & blancos & 20 & 19 & blanco & 30 & 14 & campo \\
\hline
\end{tabular}

Fuente: elaboración propia.

Como se observa, los términos más repetidos incluyen:

- Los que designan el club de fútbol: Madrid (181) -como ciudad o elipsis del nombre del club-, real (50), primera parte del nombre del 
club de fútbol, la designación equipo (59) como término general del mismo referente, blanco / s (48) referido a los colores del equipo.

- Los nombres propios de los jugadores, sobre todo de las estrellas del equipo, son muy frecuentes. Singular protagonismo tienen: Cristiano (66), acompañado por el apellido Ronaldo (18), Benzema (44), Bale (22), Modric (20), Kaká (19), Di Maria (17).

- Los que designan la organización temporal del juego y la competición: partido (66), minutos (28), liga (27), tiempo (22), parte (19), descanso (16).

- Los referidos a las jugadas y lances del juego: gol / es (74), falta (17), penalti (15).

- Los que nombran el lugar del juego: Bernabéu (36) -nombre del estadio-, defensa (20), centro (15), campo (14).

- Verbos de acción, como hizo (32), volvió (15).

Este léxico más frecuente muestra el contenido temático principal de las crónicas analizadas: el equipo del Real Madrid, sus jugadores estrella, los momentos del juego que organizan la narración, las jugadas y situaciones más destacadas, el campo donde se disputa el partido, y las acciones y movimientos realizadas por los jugadores.

\section{La composición del léxico futbolístico}

Las áreas temáticas a las que pertenece el léxico más frecuente de las crónicas deportivas analizadas coincide, grosso modo, con los «seis elementos sobre los cuales el periodista futbolístico redacta sus notas principalmente: a) el equipo como conjunto, b) un grupo específico de jugadores, c) un determinado jugador, d) el entrenador de un equipo, e) una jugada o situación en el terreno de juego y d) una situación (no jugada) fuera del terreno de juego» (Quintero Ramírez, 2016: 248).

Otros trabajos de índole léxico-semántica también han delimitado el subconjunto de los conceptos clave en la narración y comentario de un partido de fútbol a partir de glosarios futbolísticos de referencia. Tal es el caso de François (2013: 16), que determina los ocho conceptos claves de un partido de fútbol, imprescindibles para la redacción de una crónica futbolística (partido, campo, balón, gol, equipo, jugador, árbitro y espectadores), y de Calvo Córdoba (2016: 41), que identifica las cinco palabras más frecuentes en el ámbito futbolístico en lengua española (equipo, balón, jugador, fútbol, gol). 
De modo análogo, vamos a delimitar cinco términos básicos en la información futbolística, tomando en cuenta los subconjuntos de las investigaciones anteriores y los términos más repetidos en nuestro corpus. En consecuencia, analizamos detalladamente las designaciones y el modo de significar de cinco conceptos básicos que sirven de hilo conductor en las crónicas futbolísticas: equipo, jugador, partido, gol, campo.

\subsection{Equipo}

Este es un concepto central en una crónica deportiva, pues el fútbol es un deporte de equipo. Esa palabra se refiere al conjunto de los once jugadores en el campo, a los jugadores suplentes del banquillo, $\mathrm{y}$, en un sentido más amplio, al club de fútbol en su totalidad, con su reputación y características típicas.

El concepto de 'equipo' es a menudo objeto del recurso estilístico de la sustitución, por el que «el significante originario (directo) es sustituido por otro totalmente ajeno a é1 o que solo en el contexto» del relato puede comprenderse (Medina Cano, 1995: 97). Así, de forma recurrente, aparecen sinónimos del término equipo, tales como elenco, formación, institución, plantel, club y escuadra.

En nuestro corpus, para el concepto 'equipo' los términos más usados son: El Madrid (131), equipo (59), Real Madrid (50), rival / es (16), blancos (10), escuadra (6), merengue / s (4), visitantes (4), conjunto (1). Entre estas designaciones destaca la referencia exacta con el nombre del club, El Madrid y Real Madrid, aparte de los nombres de los equipos contrarios, con una función identificadora. La forma más frecuente -El Madrid- es una elipsis de el Real Madrid, y esta de el equipo del Real Madrid, que facilita su personificación para atribuirle cualidades humanas:

El Madrid no tuvo piedad de un buen Tenerife y sigue ganando. (Marca $\left.{ }^{1}, 26.09 .2009\right)$

El Madrid giró la cabeza a la derecha. (20.11.2010)

Los periodistas sustituyen el nombre del equipo por sus colores -blanco/s-, mediante el procedimiento de la metonimia. Aquí aparece el término blanco/s, y la metáfora merengue, que alude a la similitud con el color del postre de merengue, tan típico de las confiterías madrileñas del casco histórico. Funcionan tanto como sustantivo como adjetivo:

${ }^{1}$ Todos los ejemplos están tomados de la web del diario Marca. Por ello, de aquí en adelante solo se indicará la fecha de la crónica. 
Los blancos habian remontado el gol inicial de Sergio García. (16.12.2012)

Hablamos, cómo no, de los despistes de la defensa merengue en el juego aéreo. (16.03.2013)

El término equipo es neutral, sin embargo queda connotado mediante estructuras sintácticas de complemento del nombre, en las que se hace explícita la relación del equipo con su entrenador: en 10 de las concordancias se utiliza el nombre del entrenador correspondiente para designar a un equipo sin necesidad de nombrarlo. En 9 ocasiones se especifica el color del equipo -blanco- o su adscripción madridista. Finalmente otra estructura frecuente es la de oración adjetiva de relativo especificativa, para mencionar características que distinguen a este club de fútbol de otros:

El equipo de José Mourinho salió dispuesto a mandar. (21.04.2012)

Ganó el equipo blanco con muchos apuros ante el Málaga. (25.11.2017)

Lo de rendirse no va con el equipo madridista. (24.04.2010)

\subsection{Jugador}

Frente al concepto 'equipo', relativo al colectivo, 'jugador' es un concepto que hace referencia a cada uno de los individuos que integran el equipo de fútbol. La denominación de los jugadores se produce tanto a nivel individual, en torno a los jugadores destacados, como a nivel grupal, con designaciones como la zaga para hacer referencia al grupo de jugadores defensivos, o la delantera para designar a los jugadores de ataque.

A nivel individual, a los jugadores se les denomina mediante su apellido, su nombre de pila o su apodo, así como por metonimias tales como el número que portan en la camiseta, su nacionalidad o la función que desempeñan en el terreno de juego (Medina Cano, 1995: 99; Nomdedeu, 2004: 150). Según este uso periodístico, las designaciones más frecuentes en el corpus son los nombres de pila de las estrellas del Real Madrid: Cristiano (66), Benzema (44), Bale (22), etc. En segundo lugar, los jugadores han sido designados por sus posiciones tácticas y funciones sobre el terreno de juego: defensa (20), delantero (10), portero (7). Finalmente la mención de la nacionalidad ha sido otro modo de designación frecuente: portugués (8), brasileño (7), argentino (5), croata (5). 
Tabla 3. Denominaciones futbolísticas de Cristiano Ronaldo

\begin{tabular}{|l|l|l|}
\hline \multicolumn{1}{|c|}{ Nombre de pila } & \multicolumn{1}{|c|}{ Nacionalidad } & \multicolumn{1}{c|}{ Función } \\
\hline $\begin{array}{l}\text { Cristiano Ronaldo (6) } \\
\text { Cristiano (60) } \\
\text { Ronaldo (12) } \\
\text { CR9 (2), CR7 (1) }\end{array}$ & $\begin{array}{l}\text { portugués (5) } \\
\text { luso (4) }\end{array}$ & $\begin{array}{l}\text { delantero (1) } \\
\text { nueve (1) }\end{array}$ \\
\hline
\end{tabular}

Fuente: elaboración propia.

En cuanto a la onomástica, se utilizan tanto los apellidos (Benzema, Bale...) como los nombres propios simples o compuestos (Cristiano Ronaldo, Karim...), nombre y apellido (Sergio Ramos, Ángel Di María...). Llama la atención la composición formada por las iniciales de Cristiano Ronaldo (CR) y el número de su posición en el campo (7 ó 9), como si de una fórmula química o del modelo de un vehículo se tratara. En algunos casos todas estas denominaciones son reunidas en una sola frase como en:

un remate de nueve del delantero portugués... (10.02.2018).

En este caso la información es redundante, ya que se habla de un nueve para hacer referencia al centro delantero del equipo.

Para las diferentes posiciones se da la sinonimia como entre portero y meta, o entre defensa y zaga. En otros casos, aparecen hipónimos que diferencian posiciones como entre laterales y centrales, ambos dentro de la defensa, o extremo, jugador de ataque por las bandas. Así aparecen:

- portero (7), meta (5);

- defensa (20), central / es (6), zaga (2), laterales (2);

- medios (2), medio centro (1), centrocampista (1);

- delantero (10), ataque (4), extremo (1).

Por lo demás, se constata una aparición relativamente escasa de la forma neutra jugador / es (7), con un uso extensivo y general, equivalente al conjunto del equipo, que contrasta con las designaciones individuales ya comentadas:

Se puede mirar a cualquier jugador menos a Cristiano. (16.12.2012)

Pases al infinito, jugadores que no se entendían... (7.02.2016) 


\subsection{Partido}

El término partido designa un concepto fundamental en el fútbol como deporte: el 'partido de fútbol'. El DRAE define partido como un «encuentro que enfrenta a dos jugadores o a dos equipos», distinguiéndose semánticamente la idea de encontrarse para competir.

El vocablo lematizado partido tiene una frecuencia muy alta en el corpus (73), lo que no debe extrañar, ya que analizamos crónicas de partidos de fútbol. Las concordancias del término partido muestran la siguiente casuística en el corpus:

- partido + adjetivo: Aparece un adjetivo calificativo tras el sustantivo describiendo la calidad del partido. Así el partido puede ser: bravo, flojo, nuevo, plácido, terapéutico, raro, soberbio, superlativo... También aparece la estructura apositiva con sintagma nominal un partido a puerta cerrada.

- partido + proposición subordinada adjetiva:

ganó un partido que fue de fútbol y de lo que no es... (24.04.2010)

un partido que retrata mejor sus virtudes que el del Getafe (22.09.2013)

- partido + sintagma preposicional adyacente:

Ni en blanco y negro había logrado ocho goles fuera de casa en un partido de Liga. $\rightarrow$

'un partido de la competición que se juega con el sistema de liga' (20.09.2014)

El partido de Ipurua reabre un debate $\rightarrow$ 'el partido jugado en Ipurua' (4.03.2017)

- partido [Sujeto] $\rightarrow$ Predicado nominal:

el partido era ya puro fuego de artificio... (18.12.2011)

El partido resultó especialmente frustrante para Cristiano (15.03.2015)

- partido [Sujeto] $\rightarrow$ Predicado verbal:

El partido dejó en un lugar especialmente delicado a Illarramendi (4.05.2014)

El partido dio para múltiples lecturas. (15.03.2015)

- partido $[\mathrm{CD}] \leftarrow$ Verbo Predicativo:

Di María, que [...] inició el partido como suplente (16.12.2012)

le sacó el penalti que sentenció el partido. (19.11.2016)

- partido [C.C.] $\leftarrow$ Verbo Predicativo:

Entró en el partido a cuarto de hora del final (24.04.2010)

Cristiano, que venía muy tocado tras su flojo partido ante el Barcelona (18.12.2011) 


\subsection{Gol}

El concepto 'gol' alude al objetivo de este deporte de origen inglés: que el balón entre en la portería. La misma palabra española gol es un anglicismo procedente del término inglés goal, que significa precisamente 'objetivo, meta'. Los goles suelen ser los acontecimientos más importantes en un partido de fútbol. Todo el sistema táctico, la estrategia y el esfuerzo de las acciones en el terreno de juego tienen como objetivo la consecución de un gol.

Gol / es posee una frecuencia muy alta en el corpus (74). Su sinónimo es tanto / $s$ (14):

Raúl marcó en Zaragoza el único tanto que le faltaba. (24.04.2010)

Benzema zanjó la cuestión con su segundo tanto de la noche. (6.03.2011)

El análisis de concordancias muestra que, a nivel morfosintáctico, la palabra gol va acompañada de nombres propios (de un futbolista, de un equipo) para designar quién ha sido el encargado de materializar el gol. Es el caso de las construcciones gol de Benzema, gol de Cristiano, gol del Málaga, gol del Éibar. Igualmente aparece gol del luso, que funciona del mismo modo para hacer referencia al goleador sin nombrarlo directamente.

Con frecuencia se utilizan complementos adyacentes al nombre con la preposición de para designar cómo ha sido el gol. Así aparecen las construcciones gol de cabeza, gol de penalti, gol de tacón, gol del cojo, gol del empate, etc.:

Cristiano rozó el gol de la temporada ('el mejor gol del año') (26.09.2009)

Para empezar, un gol de cabeza de Peñaranda que anuló el árbitro (25.11.2017)

Los adjetivos también sirven para calificar la calidad del gol. Así se habla de gol feo, gol inicial, gol legal, gol afortunado.

En cuanto a los verbos que componen el núcleo del predicado en las concordancias con gol y tanto, en el corpus anotamos que el gol se puede hacer, marcar, meter, celebrar, remontar, añadir, rozar, negar, anular, el gol llega, decide el partido, afecta al equipo, le desarma, mata al equipo contrario, o lo espolea, evita la derrota, premia un buen partido:

Los goles llegaron mediado el primer tiempo. (6.03.2011)

Un gol de Cristiano a la contra mató al Barça... (21.04.2012)

La palabra gol tiene bastante productividad con respecto a la formación de derivados. Así, junto a gol / es (74), aparecen los derivados golear y sus formas flexivas (12), goleada / s (6), golazo (4), goleador / es (2). 
Respectivamente, golear significa 'Hacer gol al otro equipo, especialmente con reiteración', goleada 'victoria por varios goles de diferencia', golazo 'gol muy bello, espectacular', goleador 'persona que marca los goles'.

\subsection{Campo}

El último concepto que analizamos es 'campo', en un sentido amplio, como lugar donde se juega al fútbol, con designaciones relativas al césped, al estadio, o a subdivisiones de un campo de fútbol: área de penalti, círculo central, las bandas, las esquinas, etc. Igualmente, se distinguen diferentes líneas en el terreno de juego: la línea de fondo, de gol, de medio campo, de banda, etc.

La distribución en el corpus de los términos relativos al concepto 'campo' es la siguiente: Bernabéu (29), Santiago Bernabéu (8), campo (14), estadio (8), césped (5), territorio (2), pradera (1), terreno (1). Además, aparecen las subdivisiones del terreno de juego: área (14), banda / s (6), córner (4), línea (2). El procedimiento más utilizado es el de designar el estadio por su nombre propio: Santiago Bernabéu.

El nombre (Santiago) Bernabéu alude, por elipsis, tanto al estadio de fútbol, al terreno de juego, como, por metonimia, al público que presencia el partido y que anima al equipo del Real Madrid. Así, en su primera acepción, se visita el Bernabéu, sirve de fondo al partido, es un recinto con puertas, etc. Se habla de la pradera del Bernabéu y del césped del Bernabéu, pero también se dice que un equipo pisa el Bernabéu. No obstante, con frecuencia el Bernabéu se identifica con el público asistente a los partidos, con los hinchas y seguidores del equipo madridista, y por eso adquiere cualidades humanas. Así se dice que el Bernabéu se encandila con buenas jugadas, que perdona a un jugador, que un jugador tiene enamorado al Bernabéu, que el Bernabéu es un estadio exigente, está iracundo, otorga su aprobación y que reacciona como un solo ser cuando ovaciona, silba, levanta un castigo, etc.:

En campo propio, volvió Pepe, perdonado por el Bernabéu. (26.09.2009)

El Athletic acabó pisoteado en la pradera del Santiago Bernabéu. (20.11.2010)

... tiene enamorado al Bernabéu, estadio exigente que valora el esfuerzo y el no rendirse. (20.11.2010) 


\section{Conclusiones}

En este artículo hemos analizado 18 crónicas de la versión digital del diario Marca, de partidos del Real Madrid entre 2009 y 2018. Tanto el diario Marca -el más leído de España- como el Real Madrid -el club más laureado de la historia del fútbol- son dos instituciones con una gran repercusión social.

El análisis léxico, mediante frecuencias y concordancias, ha permitido identificar los conceptos tratados en estas crónicas en relación con el Real Madrid y con el deporte del fútbol, y de qué manera se representan estos conceptos desde el punto de vista léxico y semántico. El análisis del léxico más frecuente descubre que las crónicas de los partidos de fútbol del Real Madrid giran en torno al equipo como club e institución, a sus jugadores estrella, al campo donde se disputa el partido, y a las acciones y jugadas más destacadas.

La segunda parte del análisis ha consistido en un análisis detallado de las designaciones y el modo de significar de los cinco conceptos más básicos del fútbol, según las frecuencias léxicas de nuestro corpus, que coinciden, grosso modo, con estudios similares. Los conceptos analizados y los términos de referencia han sido equipo, jugador, partido, gol, campo, que sirven de hilo conductor de las crónicas seleccionadas. Gracias a este análisis se puede percibir la productividad combinatoria de los términos elegidos, los valores semánticos de estas palabras, así como la variación léxica para designar los cinco conceptos con más peso en el corpus analizado.

\section{Bibliografía}

A.I.M.C., ASOCIACIÓN PARA LA INVESTIGACIÓN DE MEDIOS DE COMUNICACIÓN (2010). Resumen general de resultados EGM, Estudio General de Medios, $3^{\circ}$ Año Móvil 2010. <https://www.aimc.es/a1mc-c0nt3nt/ uploads/2010/09/internet309.pdf $>$, fecha de consulta: 10 de noviembre de 2018.

A.I.M.C., ASOCIACIÓN PARA LA INVESTIGACIÓN DE MEDIOS DE COMUNICACIÓN (2018). Resumen general de resultados EGM, Estudio General de Medios, $2^{\circ}$ Año Móvil 2018. <https://www.aimc.es/a1mc-c0nt3nt/ uploads/2018/04/internet118.pdf>, fecha de consulta: 10 de noviembre de 2018. 
ÁLVAREZ PEDROSA, F. (2017). La crónica en el diario Marca antes y después de Internet. Análisis de la cobertura de la selección española de fútbol (1984-2008). Tesis doctoral. Valencia: Universidad CEU Cardenal Herrera.

CALVO CÓRDOBA, A. (2016). Análisis del léxico futbolístico y su tratamiento en la prensa general en inglés y en español. Trabajo fin de grado. Valladolid: Universidad de Valladolid.

FRANÇOIS, K. (2013). Sobre algunas particularidades léxico-semánticas del lenguaje del fútbol. Un análisis contrastivo: español vs. inglés. Tesis de maestría. Gante: Universidad de Gante.

HERNÁNDEZ ALONSO, N. (2003). El lenguaje de las crónicas deportivas. Madrid: Cátedra.

MARCA (2009-2018). Hemeroteca. <http://www.marca.com/hemeroteca/2018>, fecha de consulta: 13 de noviembre de 2018.

MEDINA CANO, F. (1995). «Los narradores deportivos y sus epopeyas cotidianas», Estudios sobre las Culturas Contemporáneas, Vol. 1, n² 2, 69-106.

NARANJO DE ARCOS, A. (2011). Tratamiento de la información deportiva en la prensa: la crónica como género prevalente. El caso de los encuentros de fútbol entre Real Madrid y F.C. Barcelona. Tesis doctoral. Málaga: Servicio de publicaciones de la Universidad de Málaga.

NOMDEDEU RULL, A. (2004). Terminología del fútbol y los diccionarios: elaboración de un diccionario de especialidad para el gran público. Tesis doctoral. Barcelona: Universidad Autónoma de Barcelona.

QUINTERO RAMÍREZ, S. (2016). «Estructuras sintácticas y léxicas para designar los elementos del evento futbolístico», Literatura y Lingüística, 34, 245264.

ULRICH, W. (2002). Wörterbuch linguistische Grundbegriffe, 5. a ed., Berlin, Stuttgart: Gebrüder Borntraeger. 